ORIGINAL ARTICLE

\title{
Emergency ultrasound in the acute assessment of haemothorax
}

\author{
A Brooks, B Davies, M Smethhurst, J Connolly
}

Emerg Med J 2004;21:44-46

See end of article for authors' affiliations

.....................

Correspondence to: Mr A Brooks, Department of Surgery, Queen's

Medical Centre

Nottingham NG'7 2UH,

UK; adambrooks@

btopenworld.com

Accepted for publication 7 April 2003
Aims: To evaluate thoracic ultrasound for the detection of haemothorax in patients with thoracic trauma against established investigations.

Methods: Thoracic ultrasound was performed as an extension of the standard focused assessment with sonography for trauma (FAST) protocol used at the Queen's Medical Centre for the assessment of adult patients with torso trauma. Fluid was sought in both pleural cavities using a hand portable ultrasound system by one of two non-radiologists trained in FAST. Findings were compared against subsequent investigations/procedures performed at the discretion of the attending emergency physician-supine chest radiography, intercostal drain, computed tomography, or thoracotomy. The sensitivity of the technique and the time taken to diagnosis for each investigation were recorded.

Results: Sixty one patients, 54 (89\%) after blunt trauma, underwent thoracic ultrasound evaluation during the study. Twelve patients had a haemothorax detected by ultrasound and confirmed by computed tomography or by tube thoracostomy. Four haemothoraces detected on ultrasound were not apparent on trauma chest radiography. There were 12 true positives, 48 true negatives, no false positives, and one false negative scan. The sensitivity of ultrasound was $92 \%$ and specificity $100 \%$ with a positive predictive value of $100 \%$ and negative predictive value $98 \%$ for the detection of haemothorax after trauma.

Conclusions: Emergency ultrasound of the chest performed as part of the primary survey of the traumatised patient can rapidly and accurately diagnose haemothorax and is a valuable tool to augment the immediate clinical assessment of these patients.
F ocused assessment with sonography for trauma (FAST) by trained non-radiologists has been shown to provide an accurate ${ }^{12}$ and rapid evaluation ${ }^{3}$ of the trauma patient for haemoperitoneum therefore augmenting clinical decision making. Standard FAST protocols evaluate four regions (pericardial, perihepatic, perisplenic, and pelvis) however it is technically feasible and comparatively simple to expand the scanned areas to formally assess the chest for haemothorax. This result may be available before the standard trauma supine chest radiography and could potentially be more accurate permitting early and precise definition of the location of bleeding in the cardiovascularly unstable trauma patient.

The objective of the study was to evaluate the accuracy of thoracic ultrasound for the detection of haemothorax after thoracic trauma against established investigations in a British emergency department.

\section{METHODS}

A prospective study was performed over a six month period in the emergency department of Queen's Medical Centre, Nottingham, UK. Adult patients triaged to the resuscitation room with thoracic trauma were enrolled if one of the investigators were available. The study was conducted in accordance with the ethical approval obtained from the Queen's Medical Centre Ethics Committee reference GS040102.

FAST was performed on the supine trauma patient in parallel with the primary survey, which proceeded unhindered. In addition to the four conventional FAST views oblique views were obtained to visualise both hemithoraces for the presence of free intrapleural fluid. Fluid detected either in the chest or abdomen was reported to the trauma team leader at the time, as required by the ethics committee.
All chest ultrasounds were performed and interpreted by one of two non-radiologist emergency sonographers (consultant emergency physician, general surgery specialist registrar), each of whom had in excess of 50 FAST scans experience. A dedicated emergency department hand portable Sonosite 180 ultrasound system with a 4-2 $\mathrm{mHz} 15 \mathrm{~mm}$ curved array probe was used for all scans.

Ultrasound findings were compared against the results of the supine chest radiograph, as reported by the trauma team or the presence of blood drained from the chest when an intercostal drain was placed. When a contrast enhanced computer tomography (CT) scan was organised by the team for the assessment of abdominal or thoracic injuries, the ultrasound findings were compared with the results of the scan as reported by the on call radiologist. The times that the results from the ultrasound, chest radiograph, or CT were available to the trauma team leader were compared.

\section{RESULTS}

Sixty one patients, 54 (89\%) after blunt trauma, underwent thoracic ultrasound evaluation during the study. Twelve patients had a haemothorax on ultrasound scanning, eight were confirmed by chest radiograph or tube thoracostomy that drained blood, or both. There were no false positive ultrasounds scans. Figure 1 provides a summary of the results. Tables 1 and 2 give a detailed breakdown of the results for blunt (sensitivity 90\%, specificity 100\%) and penetrating trauma (sensitivity $100 \%$, specificity $100 \%$ ).

A single false negative scan was reported in a pedestrian hit by a car resulting in a subdural haematoma and facial

Abbreviations: FAST, focused assessment with sonography for trauma; $\mathrm{CT}$, computed tomography 


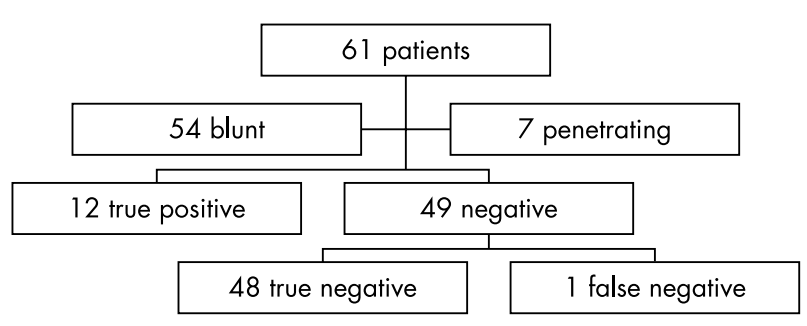

Figure 1 Results profile.

injuries. Supine chest radiography was reported as showing small bilateral effusions that were treated conservatively. Views were incomplete in three patients because of surgical emphysema from associated pneumothoraces and were excluded from analysis; none had haemothorax diagnosed on the chest radiograph.

Four traumatic effusions detected by ultrasound were confirmed by CT, but were not initially detected on the supine trauma chest radiograph. Three were clinically not significant and were managed by observation without complication, while one had an intercostal drain that drained about $100 \mathrm{ml}$ of blood.

Overall, thoracic ultrasound had a sensitivity of $92 \%$ and specificity of $100 \%$ for the detection of haemothorax after trauma. The accuracy of the technique was $98 \%$, with a positive predictive value of $100 \%$ and negative predictive value of $98 \%$.

In $42(84 \%)$ cases, the ultrasound result was available to the trauma team before the plain radiographs.

\section{DISCUSSION}

FAST has become accepted as the initial abdominal investigation of choice in trauma patients in many institutions. Positive results in unstable patients permit early decision making, appropriate triage, and management. Extending this modality routinely to the diagnosis of haemothorax would potentially further improve the evaluation of haemodynamic instability during the circulation assessment of trauma resuscitation.

We have evaluated the technique of thoracic trauma ultrasound when performed by non-radiologists in the UK, using a number of comparators of which plain radiography was one, and shown that ultrasound can rapidly and accurately diagnose haemothorax in trauma, providing a sensitive and specific tool to augment clinical examination and assessment of the patient in the circulation phase of the resuscitation. This study has shown that thoracic ultrasound is at least comparable to a supine trauma chest radiograph for the detection of haemothorax, but this should not be interpreted as a proposal for replacing the supine chest radiograph as the plain films provide valuable detail on other injuries, although Bokhari ${ }^{4}$ has brought into question the value of obtaining a chest radiograph in blunt trauma, as they found that it added little information to a normal physical examination in a stable patient.

The value of thoracic ultrasound has been assessed previously in work undertaken in North America, however the technique is not widely practised especially in the United Kingdom. Ma et al ${ }^{5}$ found that ultrasound was comparable to chest radiograph in detecting haemothorax and sensitivities for the detection of haemothorax of $81 \%$ to $96 \%^{5-7}$ have been reported. Sisley showed that the performance time for ultrasound was significantly shorter than portable chest radiograph with both techniques having comparable sensitivity. ${ }^{8}$ Ultrasound has also been reported to detect smaller quantities of fluid than chest radiography and as little as $20 \mathrm{ml}$ of pleural fluid can be detected on ultrasound ${ }^{6}$
Table 1 Details of blunt trauma results

\begin{tabular}{llc}
\hline Blunt trauma & FAST positive & FAST negative \\
\hline Actual positive & 9 & 1 \\
Actual negative & 0 & 44 \\
\hline
\end{tabular}

compared with $50-100 \mathrm{ml}$ and $175 \mathrm{ml}^{9}$ for upright and supine chest radiographs respectively. Diaphragmatic injury can also be assessed using this technique to look for ipsilateral haemoperitoneum and haemothorax from which diaphragmatic injury can be inferred in cases of penetrating thoracoabdominal trauma. ${ }^{10}$ Ultrasound techniques have also been developed to assess for pneumothorax in trauma and validated against chest radiograph with a $95 \%$ sensitivity, ${ }^{11}$ two pneumothoraces were not diagnosed as surgical emphysema obscured the view. Dulchavsky's $\mathrm{s}^{11}$ study did identify one pneumothorax that was not visible on the initial chest radiograph, however the sensitivity of ultrasound against CT for small pneumothoraces not visible on the chest radiograph has not yet been investigated.

There are a number of limitations to this study. There were times when the investigators were not on duty and thus a number of potential candidates may have been omitted from the study. Three patients were excluded from the analysis as surgical emphysema obscured views of the pleural space and this represents a potential drawback of the technique. Exclusion was justified as the procedure was not completed rather than inaccurate results being obtained. These patients require early trauma chest radiography. It could also be argued that the ultrasound results should not have been made available to the trauma team leader, however such disclosure was stipulated by the local ethics committee.

The initial trauma chest radiograph in the severely injured patient tends to be a supine film as most injured patients are managed, primarily, with spinal immobilisation. These can be difficult to interpret and require a greater volume of blood for diagnosis. ${ }^{6}{ }^{9}$ In this series ultrasound was more sensitive than supine chest radiograph for the detection of haemothorax as four traumatic effusions were reported on ultrasound that were not apparent on the initial trauma chest radiograph. In the United Kingdom these films tend to be interpreted initially by the attending emergency physician or surgeons as compared with radiologists who may form part of the trauma team in other parts of the world.

It has previously been suggested that ultrasonography could expedite the diagnosis and treatment of haemothorax in major trauma. ${ }^{7}$ In our series most of the ultrasound results were available considerably earlier than the chest radiograph despite overhead gantry radiograph equipment. Furthermore, interrogation of the standard four FAST and two thoracic views can be performed in a mean time of four minutes, ${ }^{5}$ improving the time to the diagnosis of bleeding in either of the two main body cavities.

\section{CONCLUSION}

Emergency ultrasound of the chest performed as part of the primary survey of the traumatised patient can rapidly and

Table 2 Details of penetrating trauma results

\begin{tabular}{lll}
\hline Penetrating trauma & FAST positive & FAST negative \\
\hline Actual positive & 3 & 0 \\
Actual negative & 0 & 4 \\
\hline
\end{tabular}


accurately diagnose haemothorax and is a valuable tool to augment the immediate clinical and radiological assessment of these patients.

\section{Authors' affiliations}

A Brooks, B Davies, Department of Surgery, Queen's Medical Centre, University Hospital, Nottingham, UK

M Smethhurst, J Connolly, Department of Accident and Emergency, Queen's Medical Centre

\section{REFERENCES}

1 Rozycki GS, Ochsner MG, Schmidt JA, et al. A prospective study of surgeonperformed ultrasound as the primary adjuvant modality for injured patient assessment. J Trauma 1995;39:492-500.

2 Dolich MO, McKenny MG, Varela JE, et al. 2576 ultrsound for blunt abdominal trauma. J Trauma 2001;50:108-12.
3 Boulanger BR, Brenneman FD, McLellan BA, et al. A prospective study of emergent abdominal sonography after blunt abdominal trauma. J Trauma 1995;39:325-30.

4 Bokhari F, Nagy K, Roberts R, et al. Prospective evaluation of the sensitivity of physical examination in chest trauma. J Trauma 2002;53:1135-8.

5 Ma OJ, Mateer JR, Ogata M, et al. Prospective analysis of a rapid trauma ultrasound examination performed by emergency physicians. J Trauma 1995;38:879-85

6 Rothlin MA, Nat R, Amgwerd M, et al. Ultrasound in blunt abdominal and thoracic trauma. J Trauma 1993;34:488-95.

7 Ma OJ, Mateer JR. Trauma ultrasound examination versus chest radiography in the detection of haemothorax. Ann Emerg Med 1997;29:312-15.

8 Sisley AC, Rozycki GS, Ballard RB et al. Rapid detection of traumatic effusion using surgeon-performed ultrasound. J Trauma 1998:44:291-7.

9 Juhl JH, ed. Disease of the pleura, mediastinum and diaphragm. In: Essentials of radiologic imaging. Philadelphia: Lippincott, 1993.

10 Ammann AM, BW, Maull KI, et al. Traumatic rupture of the diaphragm: realtime sonographic diagnosis. Am J Radiol 1983;140:915-16.

11 Dulchavsky SA, Schwarz KL, Kirkpatrick AW, et al. Prospective evaluation of thoracic ultrasound in the detection of pneumothorax. J Trauma 2001;50:201-5. 